\title{
一条合成毛苾花苷的新路线
}

\author{
胡志飞 $a, b$ 徐 鹏 $b$ 俞 风炎*,a,b \\ $\left({ }^{a}\right.$ 上海科技大学物质科学与技术学院 上海 201210) \\ $\left({ }^{b}\right.$ 中国科学院上海有机化学研究所 生命有机化学国家重点实验室 上海 200032)
}

\begin{abstract}
摘要 毛荧花苷(Acteoside)是苯丙素苷类天然产物中的代表性分子, 具有广泛的生物活性. 报道了一条新的合成毛荧 花苷的路线. 在合成中, 利用鼠李糖基邻炔基苯甲酸酯给体和 2,3,4-三差基葡萄糖硫苷在金(I)催化下的区域选择性糖苷 化, 得到关键的 $\alpha-(1 \rightarrow 3)$ 连接二糖硫苷 7-1, 通过二糖的邻炔基苯甲酸酯给体合成苯丙素苷, 进而完成了毛蒿花苷的合 成. 区域选择性的糖苷化减少了保护基操作步骤，缩短了合成路线.
\end{abstract}

关键词 毛莣花苷; 苯丙素苷; 糖苷化; 金(I)催化

\section{A New Approach to the Synthesis of Acteoside}

\author{
Hu, Zhifei ${ }^{a, b} \quad \mathrm{Xu}$, Peng $^{b} \quad \mathrm{Yu}, \mathrm{Biao}^{*, a, b}$ \\ ( ${ }^{a}$ School of Physical Science and Technology, ShanghaiTech University, Shanghai 201210) \\ $\left({ }^{b}\right.$ State Key Laboratory of Bioorganic and Natural Products Chemistry, Shanghai Institute of Organic Chemistry, Chinese \\ Academy of Sciences, Shanghai 200032)
}

\begin{abstract}
A new approach to the synthesis of acteoside, a prototypical phenylpropanoid glycoside with a variety of biological activities, has been developed. The synthesis employed a regioselective glycosylation as a key step, in which a gold(I)-catalyzed glycosylation of $p$-tolyl 6-O-acetyl-1-thio- $\beta$ - $D$-glucopyranoside with peracetyl $L$-rhamnopyranosyl ortho-alkynylbenzoate led to the desired $\alpha-(1 \rightarrow 3)$ linked disaccharide 7-1 in a satisfactory yield. The resultant disaccharide was converted into the corresponding ortho-alkynylbenzoate donor and subjected to glycosylation with aglycone, subsequent deprotection of the protecting groups furnished acteoside.

Keywords acteoside; phenylpropanoid glycoside; glycosylation; $\mathrm{Au}(\mathrm{I})$ catalysis
\end{abstract}

苯丙素苷化合物(Phenylpropanoid Glycosides, 简称 PPGs)是一类在自然界中广泛存在的糖苷化合物, 尤其 在玄参科和唇形科植物中最为常见 ${ }^{[1]}$. PPGs 的核心结构 通常是 2 -苯乙基- $\beta$ - $D$-吡喃葡萄糖苷，其 2,4 位或 6 位羟 基通过酯键连接反式的取代肉桂酰基，2，3，4 或 6 位羟 基再通过糖苷键连接 $L$-鼠李糖( Rha)、D-葡萄糖(Glc)和 $D$-木糖(Xyl)等(图 1) ${ }^{[2]}$. 因其苷元为取代的苯乙基, 亦被 称为苯乙醇苷类化合物 (Phenylethanoid glycosides, PhEGs). 由于取代单糖的种类和连接位置的不同, 以及 肉桂酰基和苯乙基上取代基团的变化, 该类化合物呈现 丰富的结构多样性.

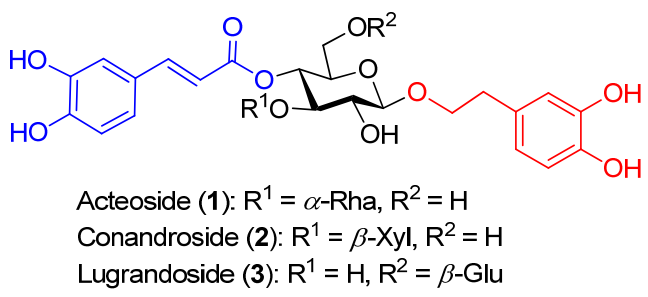

图 1 苯丙素苷的代表性结构

Figure 1 Structures of typical phenylpropanoid glycosides

毛芯花苷(Acteoside), 作为 PPGs 家族中的明星分 子, 最早由 Scarpati 等 ${ }^{[2 \mathrm{a}]}$ 在 1963 年从毛芯花属植物

\footnotetext{
* Corresponding author. E-mail: byu@sioc.ac.cn

Received April 30, 2020; revised May 13, 2020; published online May 19, 2020

Dedicated to Professor Henry N. C. Wong on the occasion of his 70th birthday.

Project supported by the National Key Research \& Development Program of China (No. 2018YFC0310900), the National Natural Science Foundation of China (No. 21621002), the Key Research Program of Frontier Sciences of Chinese Academy of Sciences (No. ZDBS-LY-SLH030), the Strategic Priority Research Program of Chinese Academy of Sciences (No. XDB20020000), and the K. C. Wong Education Foundation.

国家重点研发计划(No. 2018YFC0310900)、国家自然科学基金(No. 21621002)、中国科学院前沿科学重点研究(No. ZDBS-LY-SLH030)、中国科学院 战略性先导科技专项(B 类)(No. XDB20020000)和王宽诚教育基金会资助项目.
} 
Verbascum sinuatum 中提取得到并命名为 “Verbascoside”。直到 1968 年, Birkofer 等 ${ }^{[3]}$ 才确定了该分子的结 构, 并命名为 “Acteoside” (图 1). 迄今, Acteoside 已经 被证实是多种中药(例如肉苁蓉、地黄、大叶紫珠等)的 重要有效成分之一, 在抗氧化 ${ }^{[4]}$ 、抗炎杀菌 ${ }^{[5]}$ 、抗肿瘤 ${ }^{[6]}$ 、 抗病毒 ${ }^{[7]}$ 和保肝护肝 ${ }^{[8]}$ 等方面具有广泛的生物活性. 但 是, 其在植物中的含量低微 $(0.02 \% \sim 0.4 \%)$, 因此, 如何 通过化学方法高效合成 Acteoside 受到科学家的关注 ${ }^{[9]}$.

苯丙素苷类分子中存在 $\alpha, \beta$-不饱和酯基, 限制了糖 苷合成中常用保护基的使用，导致目前对该类天然产物 的化学合成路线存在保护基操作繁琐、步骤较多等缺 陷 ${ }^{[10-16]}$. 1999 年, Kawada 课题组 ${ }^{[10]}$ 和 van Boom 课题 组 ${ }^{[11]}$ 先后报道了 Acteoside 的全合成. 前者在合成中使 用了苄基保护酚羟基, 其脱除条件易破坏咖啡酸结构, 因此，总产率较低(最长线性 11 步，总收率 3.5\%); 而后 者虽然选取了较易脱除的保护基, 但是经历了较多保护 基的转换操作, 合成路线较长(最长线性 16 步, 总收率 $7.1 \%$ ). 为了减少保护基操作, 2017 年, Judeh 小组 ${ }^{[16 b]}$ 使 用 2-氨基乙基二苯基硼酸酯活化多羟基受体, 与卤苷给 体进行区域选择性糖苷化生成相应原酸酯, 乙酰基保护 2,4 位羟基后, 在三氟甲磺酸三甲基硅酯(TMSOTf)促进 下转化为 $1 \rightarrow 3$ 连接的二糖, 并完成了苯丙素苷 Osmanthuside- $\mathrm{B}_{6}$ 的合成. 我们拟利用葡萄糖上各个羟 基的反应活性的差别 ${ }^{[17]}$, 应用我们实验室发展的一价 金催化的糖苷化反应 ${ }^{[18]}$, 尝试区域选择性的糖苷化来 合成关键的 $\alpha-(1 \rightarrow 3)$ 连接二糖 7-1, 以完成 Acteoside 和 其它苯丙素苷类分子的高效合成.

\section{1 结果与讨论}

按照文献方法制备得到鼠李糖邻炔基苯甲酸酯给 体 $5^{[19]}$ 和 6 位差基乙酰化的葡萄糖硫苷受体 $\mathbf{6}^{[20]}$. 随后, 我们尝试在三苯基膦金(I)双(三氟甲磺酰基)亚胺盐 $\left(\mathrm{Ph}_{3} \mathrm{PAuNTf}_{2}\right)$ 催化下, 实现糖基炔酯给体 5 对 $2,3,4$-三羟 基裸露的受体 6 的区域选择性糖苷化反应(表 1). 考虑到 糖环上不同位置羟基的反应活性差异一般在低温下较 明显, 反应首先选择在一 $70{ }^{\circ} \mathrm{C}$ 下进行(Entry 1), 以 $32 \%$ 的收率得到了期望的 $\alpha-(1 \rightarrow 3)$ 糖苷化产物 7-1, 其主要 副产物为 $\alpha-(1 \rightarrow 4)$ 糖苷化二糖产物 7-2, 收率为 $5 \%$, 说 明该反应具有较好的区域选择性. 产率较低的原因是反 应不完全, 有较多的给体剩余. 为了提高给体的转化率, 我们升高反应温度至 $-30{ }^{\circ} \mathrm{C}$, 薄层色谱(TLC)监测发现 有新的副产物产生, 而在糖苷化反应基本结束后将反应 体系升至室温搅拌, 该新的副产物大部分消失, 因此, 怀疑上述副产物是原酸酯化合物, 升至室温后转化为正 常糖苷化产物或水解产物, 目标产物 7-1 的产率为 46\%
(Entry 2). 考虑到原酸酯一般可以在路易斯酸催化下转 化为正常的糖苷化产物 ${ }^{[21]}$, 分别在糖苷化反应开始和 结束后加入 0.1 equiv.的 TMSOTf, 考察催化量的路易斯 酸在反应的不同时期促进上述副产物向二糖 7-1 的转化 情况. 我们发现在反应开始即加入 TMSOTf 并不能在反 应过程中有效促进原酸酯的转化(Entry 4); 而在给体 5 基本消耗完全后升至室温再加入 TMSOTf 可以有效促 进原酸酯向 7-1 的转化(Entry 5). 最后, 以 $61 \%$ 的收率得 到了所需的 $\alpha-(1 \rightarrow 3)$ 糖苷化产物 7-1.

表 1 受体 6 与给体 5 的区域选择性糖苷化 ${ }^{a}$ Table 1 Optimization of the regioselective glycosylation of acceptor $\mathbf{6}$ with donor $\mathbf{5}$
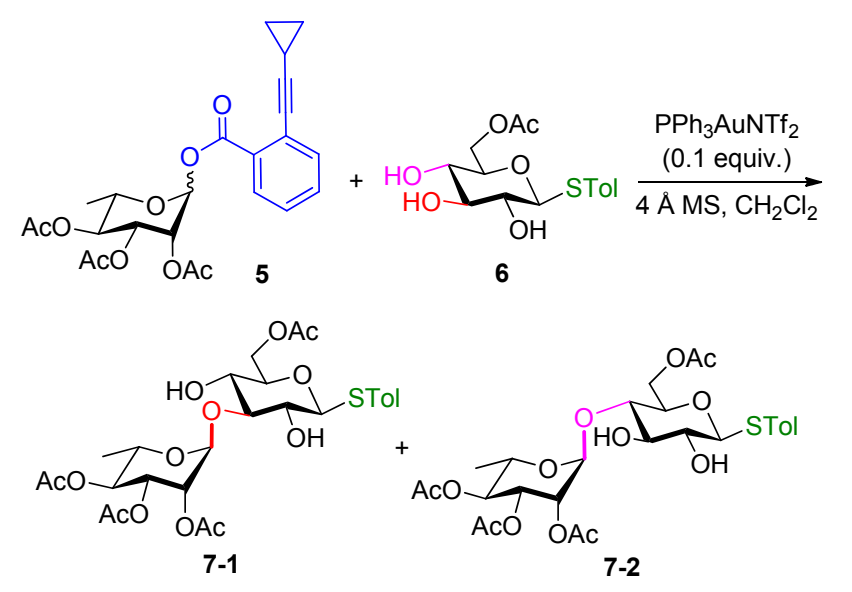

\begin{tabular}{ccccc}
\hline \multirow{2}{*}{ Entry } & \multirow{2}{*}{ Temp. $/{ }^{\circ} \mathrm{C}$} & \multirow{2}{*}{ TMSOTf/equiv. } & \multicolumn{2}{c}{ Yield $^{d} / \%$} \\
\cline { 4 - 5 } & & & $\mathbf{7 - 1}$ & $\mathbf{7 - 2}$ \\
\hline 1 & -70 & & $32 \%$ & $5 \%$ \\
2 & $-30 \sim$ r.t. & & $46 \%$ & Trace \\
3 & $-30 \sim$ r.t. & $0.1^{b}$ & $48 \%$ & Trace \\
4 & $-30 \sim$ r.t. & $0.1^{c}$ & $61 \%$ & Trace \\
\hline
\end{tabular}

${ }^{a}$ Reaction conditions: $5(0.1 \mathrm{mmol}), 6(0.1 \mathrm{mmol}), 4 \AA \mathrm{MS}(250 \mathrm{mg}), \mathrm{CH}_{2} \mathrm{Cl}_{2}$ $(5 \mathrm{~mL}), 20 \mathrm{~h} ;{ }^{b}$ TMSOTf was added at the beginning of the reaction; ${ }^{c}$ TMSOTf was added after consumption of the donor; ${ }^{d}$ Isolated yields.

在顺利得到 $\alpha-(1 \rightarrow 3)$ 糖苷化产物 7-1 后，尝试在葡 萄糖单元的 2 位选择性引入保护基(表2). 考虑到目标分 子中含有咖啡酸这个 $\alpha, \beta$-不饱和酯基结构, 尝试使用可 以正交脱除的硅基进行保护. 但是, 在实验中发现 7-1 中葡萄糖 2 位羟基位阻较大，即便使用叔丁基二甲硅基 三氟甲磺酸酯(TBSOTf)或 TMSOTf 在碱性条件下也难 以对该羟基进行硅基保护(Entries 1 4). 而且还发现氯 乙酰基也同样难以引入, 并且对 2,4 位羟基的选择性较 差. 经过初步篮选反应条件, 仅以约 30\%的收率得到期 望的产物 8 (Entries 5 7). 综合考虑后, 选择体积较小 的乙酰基进行选择性保护，在上述选择性酰化条件篎选 的基础上，使用乙酰氯在 4-二甲氨基吡啶(DMAP)促进 下以最高 $51 \%$ 的收率得到葡萄糖 2 位乙酰基保护的二糖 硫苷 9. 另外, 也尝试了直接使用咖啡酸与 7-1 上的 4 位 
羟基进行选择性酯化. 但是, 发现使用 1-乙基-3(3-二甲 基丙胺)碳二亚胺(EDCI)/二环己基碳二亚胺(DCC)等缩 合剂均无法很好地区分葡萄糖的 2,4 位羟基.

表 2 二糖 7-1 中 2 位羟基的选择性保护 ${ }^{a}$

Table 2 Selective protection of the 2-OH in 7-1

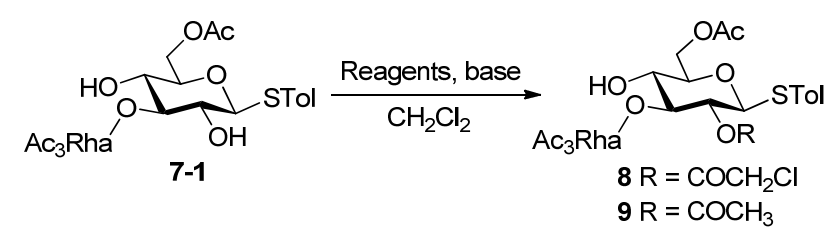

\begin{tabular}{clll}
\hline Entry & \multicolumn{1}{c}{ Reagent } & \multicolumn{1}{c}{ Base } & \multicolumn{1}{c}{ Product } \\
\hline 1 & TBSCl & Imidazole & NR \\
2 & TBSOTf & 2,6-Lutidine & Trace \\
3 & TBSOTf & NEt $_{3}$ & Trace \\
4 & TMSOTf & 2,6 -Lutidine & Trace \\
5 & ClCOCH $_{2} \mathrm{Cl}$ & $\mathrm{NEt}_{3}$ & $\mathbf{8}(23 \%)^{b}$ \\
6 & $\mathrm{ClCOCH}_{2} \mathrm{Cl}$ & Pyridine $^{b}$ & Trace \\
& & & $\mathbf{8}(35 \%)^{b}$ \\
7 & $\mathrm{ClCOCH}_{2} \mathrm{Cl}$ & DMAP & $(26 \% \sim 32 \%)$ \\
8 & $\mathrm{ClCOCH}_{3}$ & DMAP & $\mathbf{9}(51 \%)^{b}$ \\
\hline${ }^{a} \mathbf{7 - 1}(0.1 \mathrm{mmol})$, base $(0.13 \sim 0.2 \mathrm{mmol}), 0{ }^{\circ} \mathrm{C} \sim$ r.t.,, $15 \mathrm{~h}^{b}{ }^{b}$ Isolated yields.
\end{tabular}

接下去, 按照文献方法制备了烯丙基保护的咖啡酸 片段 $\mathbf{1 0}^{[22]}$, 并尝试其与二糖 9 中的葡萄糖 4 位羟基在缩 合条件下酯化. 首先使用常用的 EDCI 作为缩合剂, 发 现该反应收率较低, 有较多的杂质生成, 可能是由于乙 酰基的迁移所造成. 改用 DCC 作缩合剂, 可有效抑制
该类副产物的生成，以 $79 \%$ 的收率得到二糖 11 . 随后， 制备了烯丙基保护的苯乙醇片段 $12^{[23]}$, 并尝试其与硫 苷 11 的糖苷化反应. 使用常规的 $N$-碘代丁二酰亚胺 (NIS)/路易斯酸活化条件时，在低温 $\left(-30 \sim-20{ }^{\circ} \mathrm{C}\right)$ 下, 该二糖硫苷不易活化; 而升高反应温度，则导致硫苷的 降解. 使用 1-(苯基磺酰基)哌啶(BSP)/三氟甲磺酸酐 $\left(\mathrm{Tf}_{2} \mathrm{O}\right)^{[24]}$ 作为活化剂时, 给体水解严重, 糖苷化的产率 不理想. 因此, 尝试将硫苷转变为相应的邻炔基苯甲酸 酯给体. 尝试使用 $N$-溴代丁二酰亚胺(NBS)或 NIS 水解 硫苷，却发现化合物 11 基本不反应; 额外加入三氟乙酸 则导致反应混乱. 最终, 使用 $\mathrm{BSP} / \mathrm{Tf}_{2} \mathrm{O}$ 反应体系成功水 解了二糖硫苷 11; 随后, 将其转化成邻炔基苯甲酸酯给 体，并直接与苯乙醇 12 进行金催化糖苷化反应，顺利得 到化合物 13 (Scheme 1).

最后，只需要进行烯丙基和乙酰基这两种保护基的 脱除便能得到目标产物 Acteoside. 考虑到脱除烯丙基 所使用的钯试剂和三苯基膦配体可能不易与终产物分 离, 选择先烯丙基后乙酰基的脱除顺序. 在实际操作中, 化合物 13 在四三苯基膦钯 $\left[\left(\mathrm{PPh}_{3}\right)_{4} \mathrm{Pd}\right]$ 的作用下，以 $80 \%$ 的收率顺利脱除四个烯丙基得到化合物 14. 最后，以甲 醇和二氯甲烷的混合溶剂，化合物 14 在甲胺条件下脱 除所有乙酰基 ${ }^{[25]}$ ，以 $42 \%$ 的收率得到目标产物 Acteoside (Scheme 1). 乙酰基脱除这一步反应产率并不 理想, 主要是因为该条件导致部分咖啡酸支链断裂; 除 此之外, 文献报道类似的碱性条件可能导致 $4 \rightarrow 6$ 位酰 基迁移，收率也不理想 ${ }^{[26]}$.

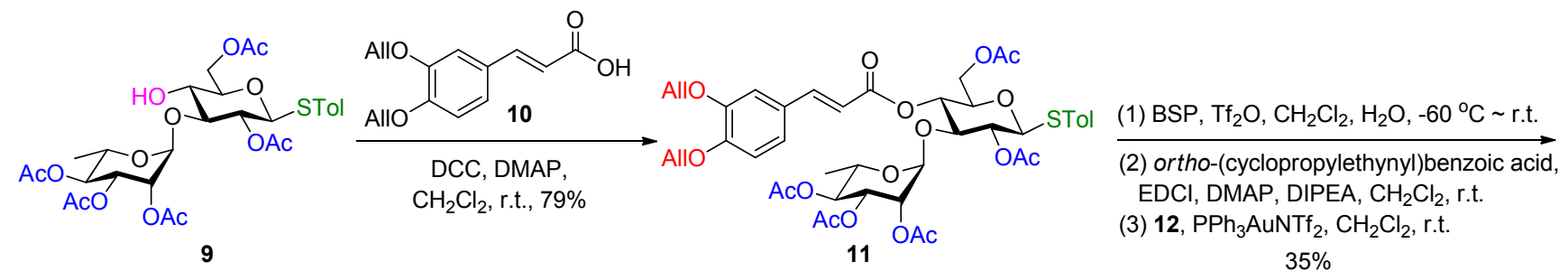

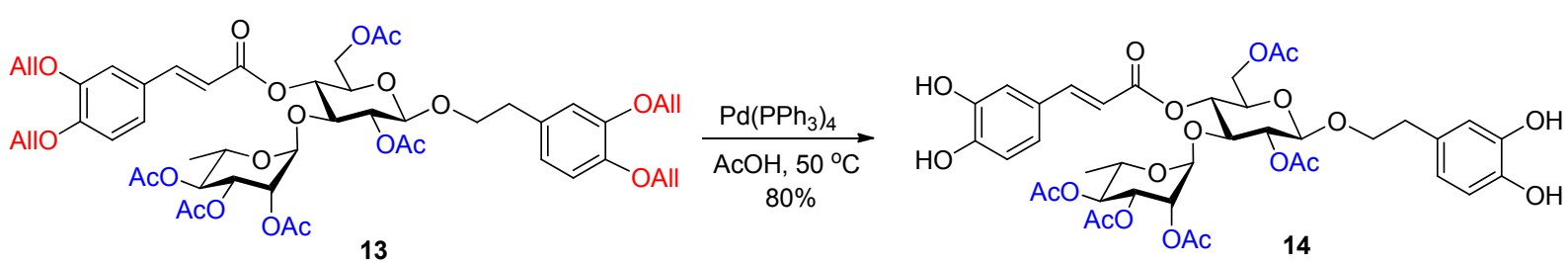

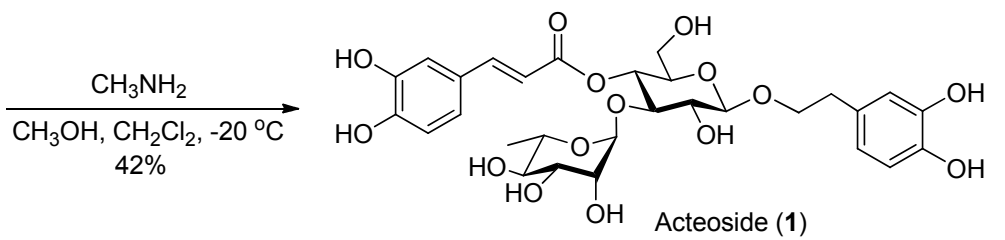<smiles>OCCc1ccc(O)c(O)c1</smiles>

图式 1 Acteoside 的合成 Scheme 1 Synthesis of acteoside 


\section{2 结论}

发展了一条合成毛芯花苷(Acteoside)的新路线, 以 已知化合物 5 和 6 为起始原料, 最长线性步骤为 8 步, 总 产率为 3\%. 其中, 关键步骤是 $\mathrm{PPh}_{3} \mathrm{AuNTf}_{2}$ 催化下的全 乙酰化鼠李糖邻炔基苯甲酸酯给体 5 与 2,3,4-三羟基葡 萄糖硫苷 6 的区域选择性糖苷化反应, 以较好的收率得 到 $\alpha-(1 \rightarrow 3)$ 二糖 7-1. 该合成路线减少了保护基操作, 缩 短了合成步数, 并可以用于其它苯丙素苷类化合物的合 成.

\section{3 实验部分}

\section{1 仪器与试剂}

核磁共振数据由 BrukerAV-400 和 Agilent-500 型核 磁共振仪测定 (以 TMS 作为内标); 高分辨质谱由 Agilent 6224 TOF LC/MS 和 Agilent TOF LC/MS 12606230 型仪器测定; 比旋光度由 Anton Paar MCP 5500 旋 光仪测定, 光源为钠光灯, $589 \mathrm{~nm}$; 薄层色谱监测采用 质量分数为 $10 \%$ 的浓硫酸乙醇加热显色或是 UV254 nm 显色. 未特别注明的商业化试剂并未进行进一步纯化. 常用的溶剂使用微波炉活化的 $4 \AA$ 分子篮进行干燥.

\section{2 化合物合成}

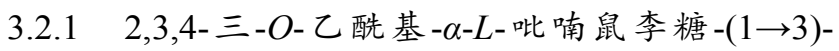
6-O-乙酰基- $\beta-D$ - 吡喃葡萄糖对甲苯硫酚苷(7-1) 和 $2,3,4$-三- $O$-乙酰基- $\alpha-L$ - 吡喃鼠李糖-( $1 \rightarrow 4)-6-O$-乙酰 基- $\beta$ - $D$-吡喃葡萄糖对甲苯硫酚苷(7-2)的合成

将鼠李糖给体 5 (46 mg, 0.1 mmol)和硫苷 6 (33 mg, $0.1 \mathrm{mmol})$ 溶于干燥的二氯甲烷 $(4 \mathrm{~mL})$ 中, 加入活化好的 $4 \AA \mathrm{MS}(250 \mathrm{mg})$, 氩气保护下室温搅拌 $0.5 \mathrm{~h}$. 反应体系 降温至 $-30{ }^{\circ} \mathrm{C}$, 通过注射器逐滴加入 $\mathrm{PPh}_{3} \mathrm{AuNTf}_{2}$ 的二 氯甲烷溶液 $(0.01 \mathrm{~mol} / \mathrm{L}, 1 \mathrm{~mL})$. 在一 $30{ }^{\circ} \mathrm{C}$ 下反应 $20 \mathrm{~h}$, 加入 TMSOTf $(1.8 \mu \mathrm{L}, 0.01 \mathrm{mmol})$. 体系升温至室温反 应 $3 \mathrm{~h}$, 加入 $\mathrm{Et}_{3} \mathrm{~N}$ 淬灭反应, 反应液经硅藻土过滤并浓 缩, 柱层析 $[V$ (石油醚) $: V($ 乙酸乙酯 $)=4: 1$ 至 $2: 1)$ 得 白色泡沫状固体 7-1 (37 mg, 产率 61\%), 并分离得到少 量的 7-2 $(<5 \%)$.

7-1: $[\alpha]_{\mathrm{D}}^{25}-72.2\left(c \quad 0.44, \mathrm{CHCl}_{3}\right) ;{ }^{1} \mathrm{H}$ NMR $(500$ $\left.\mathrm{MHz}, \mathrm{CDCl}_{3}\right) \delta: 7.46 \sim 7.41(\mathrm{~m}, 2 \mathrm{H}), 7.14(\mathrm{~d}, J=7.9 \mathrm{~Hz}$, 2H), $5.34(\mathrm{dd}, J=3.4,1.9 \mathrm{~Hz}, 1 \mathrm{H}), 5.28$ (dd, $J=10.0,3.4$ $\mathrm{Hz}, 1 \mathrm{H}), 5.18$ (d, $J=1.6 \mathrm{~Hz}, 1 \mathrm{H}), 5.07$ (t, $J=9.9 \mathrm{~Hz}, 1 \mathrm{H})$, 4.47 (dd, $J=12.2,4.4 \mathrm{~Hz}, 1 \mathrm{H}), 4.41$ (d, $J=9.7 \mathrm{~Hz}, 1 \mathrm{H})$, $4.36(\mathrm{dd}, J=12.1,2.1 \mathrm{~Hz}, 1 \mathrm{H}), 4.19$ (dq, $J=12.5,6.1 \mathrm{~Hz}$, $1 \mathrm{H}), 3.60$ (t, $J=8.7 \mathrm{~Hz}, 1 \mathrm{H}), 3.47$ (ddd, $J=9.9,4.3,2.1$ $\mathrm{Hz}, 1 \mathrm{H}), 3.42 \sim 3.34(\mathrm{~m}, 2 \mathrm{H}), 2.37(\mathrm{~s}, 3 \mathrm{H}), 2.16(\mathrm{~s}, 3 \mathrm{H})$, 2.15 (s, 3H), 2.05 (s, 3H), 2.00 (s, 3H), 1.22 (d, $J=6.3 \mathrm{~Hz}$,
$3 \mathrm{H}) ;{ }^{13} \mathrm{C}$ NMR (126 MHz, $\left.\mathrm{CDCl}_{3}\right) \delta: 171.6,170.2,170.1$, $169.9,138.7,133.7,129.8,127.2,98.5,88.2,84.1,78.0$, 71.7, 70.9, 69.7, 69.0, 68.5, 67.0, 63.3, 21.2, 20.9, 20.9, 20.8, 20.7, 17.4; HRMS (ESI) calcd for $\mathrm{C}_{27} \mathrm{H}_{36} \mathrm{O}_{13} \mathrm{SNa}$ $[\mathrm{M}+\mathrm{Na}]^{+}$623.1769, found 623.1773 .

7-2: $[\alpha]_{\mathrm{D}}^{25}-32.4\left(\right.$ c $\left.0.24, \mathrm{CHCl}_{3}\right) ;{ }^{1} \mathrm{H}$ NMR $(500$ $\left.\mathrm{MHz}, \mathrm{CDCl}_{3}\right) \delta: 7.45 \sim 7.41(\mathrm{~m}, 2 \mathrm{H}), 7.12(\mathrm{~d}, J=7.9 \mathrm{~Hz}$, 2H), $5.26(\mathrm{dd}, J=9.9,3.3 \mathrm{~Hz}, 1 \mathrm{H}), 5.13 \sim 5.06(\mathrm{~m}, 2 \mathrm{H})$, $4.87(\mathrm{~d}, J=1.7 \mathrm{~Hz}, 1 \mathrm{H}), 4.45$ (d, $J=9.8 \mathrm{~Hz}, 1 \mathrm{H}), 4.42$ (dd, $J=13.2,2.9 \mathrm{~Hz}, 1 \mathrm{H}), 4.27(\mathrm{dd}, J=12.2,4.4 \mathrm{~Hz}, 1 \mathrm{H}), 4.13$ (dq, $J=12.4,6.2 \mathrm{~Hz}, 1 \mathrm{H}), 3.76$ (d, $J=1.8 \mathrm{~Hz}, 1 \mathrm{H}, \mathrm{OH})$, $3.64 \sim 3.56(\mathrm{~m}, 2 \mathrm{H}), 3.50 \sim 3.45(\mathrm{~m}, 1 \mathrm{H}), 3.31$ (ddd, $J=$ 10.4, 8.8, 2.0 Hz, 1H), 2.60 (d, J=1.9 Hz, 1H, OH), 2.35 (s, 3H), 2.14 (s, 3H), 2.09 (s, 3H), $2.05(\mathrm{~s}, 3 \mathrm{H}), 2.00(\mathrm{~s}$, $3 \mathrm{H}), 1.24(\mathrm{~d}, J=6.3 \mathrm{~Hz}, 3 \mathrm{H}) ;{ }^{13} \mathrm{C} \mathrm{NMR}\left(126 \mathrm{MHz}, \mathrm{CDCl}_{3}\right)$ $\delta: 170.6,170.0,169.9,169.8,138.8,133.8,129.7,127.0$, $98.8,87.5,80.1,76.5,76.1,71.5,70.6,70.0,68.3,67.9$, 62.5, 21.2, 20.8, 20.8, 20.8, 20.7, 17.3; HRMS (ESI) calcd for $\mathrm{C}_{27} \mathrm{H}_{36} \mathrm{O}_{13} \mathrm{SNa}[\mathrm{M}+\mathrm{Na}]^{+}$623.1769, found 623.1766 .

3.2.2 2,3,4-三- $O$-乙酰基- $\alpha-L$ - 吡喃鼠李糖-( $1 \rightarrow 3)-6$ $O$-乙酰基- $2-O$ - 氯乙酰基- $\beta-D$-吡南葡萄糖对甲苯硫酚 甘 $(8)$ 的合成

将化合物 7-1 (300 mg, 0.50 mmol)和 DMAP (73 $\mathrm{mg}, 0.60 \mathrm{mmol})$ 溶于干燥的二氯甲烷 $(5 \mathrm{~mL})$ 中, 氩气保 护, $0{ }^{\circ} \mathrm{C}$ 下逐滴加入氯乙酰氯( $(186 \mu \mathrm{L}, 2.50 \mathrm{mmol})$, 保持 $0{ }^{\circ} \mathrm{C}$ 反应 $1 \mathrm{~h}$ 后升至室温反应 $10 \mathrm{~h}$, 乙酸乙酯稀释, 反 应液用饱和食盐水洗, 有机相用无水 $\mathrm{Na}_{2} \mathrm{SO}_{4}$ 干燥, 过 滤, 浓缩, 柱层析 $[V$ (石油醚 $): V($ 乙酸乙酯 $)=6: 1$ 至 4: 1]得白色泡沫状固体 $8(120 \mathrm{mg}$, 产率 $35 \%)$ : $[\alpha]_{\mathrm{D}}^{25}$ -43.6 (c 0.35, $\left.\mathrm{CHCl}_{3}\right)$; ${ }^{1} \mathrm{H}$ NMR $\left(500 \mathrm{MHz}, \mathrm{CDCl}_{3}\right) \delta$ : $7.41 \sim 7.37(\mathrm{~m}, 2 \mathrm{H}), 7.11(\mathrm{~d}, J=7.9 \mathrm{~Hz}, 2 \mathrm{H}), 5.21(\mathrm{dd}, J=$ $10.1,3.4 \mathrm{~Hz}, 1 \mathrm{H}), 5.06(\mathrm{t}, J=9.9 \mathrm{~Hz}, 1 \mathrm{H}), 5.00$ (dd, $J=$ $3.4,1.9 \mathrm{~Hz}, 1 \mathrm{H}), 4.95$ (t, $J=9.6 \mathrm{~Hz}, 1 \mathrm{H}), 4.90$ (d, $J=1.9$ $\mathrm{Hz}, 1 \mathrm{H}), 4.56$ (d, $J=10.1 \mathrm{~Hz}, 1 \mathrm{H}), 4.47$ (dd, $J=12.2,3.3$ $\mathrm{Hz}, 1 \mathrm{H}), 4.36$ (dd, $J=12.2,1.4 \mathrm{~Hz}, 1 \mathrm{H}), 4.30$ (d, $J=14.8$ $\mathrm{Hz}, 1 \mathrm{H}), 4.20$ (d, $J=14.8 \mathrm{~Hz}, 1 \mathrm{H}), 4.15$ (dt, $J=9.7,6.2$ $\mathrm{Hz}, 1 \mathrm{H}), 3.66(\mathrm{dt}, J=8.9,4.1 \mathrm{~Hz}, 1 \mathrm{H}), 3.49 \sim 3.42(\mathrm{~m}, 3 \mathrm{H}$, $1 \mathrm{OH}), 2.34(\mathrm{~s}, 3 \mathrm{H}), 2.16(\mathrm{~s}, 3 \mathrm{H}), 2.13(\mathrm{~s}, 3 \mathrm{H}), 2.04(\mathrm{~s}, 3 \mathrm{H})$, 1.99 (s, 3H), 1.19 (d, $J=6.3 \mathrm{~Hz}, 3 \mathrm{H}) ;{ }^{13} \mathrm{C}$ NMR (126 MHz, $\left.\mathrm{CDCl}_{3}\right) \delta: 171.7,170.2,169.9,169.8,166.1,138.6,133.7$, 129.6, 127.8, 98.6, 85.7, 84.4, 77.9, 72.1, 70.7, 70.2, 68.9, 68.3, 67.6, 62.9, 40.7, 21.2, 20.9, 20.9, 20.8, 20.7, 17.3; HRMS (ESI) calcd for $\mathrm{C}_{29} \mathrm{H}_{37} \mathrm{ClO}_{14} \mathrm{SNa}[\mathrm{M}+\mathrm{Na}]^{+}$ 699.1485 , found 699.1490 . 
3.2.3 2,3,4-三- $O$-乙酰基- $\alpha-L$ - 吡喃鼠李糖-( $(1 \rightarrow 3)$ $2,6-$ 二- $O$-乙酰基- $\beta$ - $D$ - 吡喃葡萄糖对甲苯硫酚苷(9)的 合成

以化合物 7-1 (330 mg, $0.55 \mathrm{mmol})$ 为原料, 按照化 合物 8 的合成方法制备得到白色化合物 $9(160 \mathrm{mg}$, 产率 48\%): $[\alpha]_{\mathrm{D}}^{25}-43.7$ ( c $\left.0.31, \mathrm{CHCl}_{3}\right) ;{ }^{1} \mathrm{H}$ NMR $(500 \mathrm{MHz}$, $\left.\mathrm{CDCl}_{3}\right) \delta: 7.41 \sim 7.38(\mathrm{~m}, 2 \mathrm{H}), 7.12(\mathrm{~d}, J=7.9 \mathrm{~Hz}, 2 \mathrm{H})$, $5.23(\mathrm{dd}, J=10.0,3.3 \mathrm{~Hz}, 1 \mathrm{H}), 5.11 \sim 5.05(\mathrm{~m}, 2 \mathrm{H}), 4.95$ (t, $J=9.6 \mathrm{~Hz}, 1 \mathrm{H}), 4.89$ (d, $J=2.0 \mathrm{~Hz}, 1 \mathrm{H}), 4.55$ (d, $J=$ $10.1 \mathrm{~Hz}, 1 \mathrm{H}), 4.44 \sim 4.37(\mathrm{~m}, 2 \mathrm{H}), 4.20 \sim 4.12(\mathrm{~m}, 1 \mathrm{H})$, $3.61(\mathrm{dt}, J=9.0,4.0 \mathrm{~Hz}, 1 \mathrm{H}), 3.51 \sim 3.45(\mathrm{~m}, 3 \mathrm{H}, 1 \mathrm{OH})$, 2.35 (s, 3H), 2.19 (s, 3H), 2.15 (s, 6H), 2.05 (s, 3H), 1.99 (s, 3H), $1.22(\mathrm{~d}, J=6.3 \mathrm{~Hz}, 3 \mathrm{H}) ;{ }^{13} \mathrm{C}$ NMR $(126 \mathrm{MHz}$, $\left.\mathrm{CDCl}_{3}\right) \delta: 171.5,170.0,169.9,169.7,169.5,138.3,133.3$, $129.6,128.4,98.8,86.1,85.4,77.8,70.7,70.3,70.0,69.1$, 68.5, 67.6, 63.1, 21.1, 20.9, 20.9, 20.8, 20.7, 20.6, 17.3; HRMS (ESI) calcd for $\mathrm{C}_{29} \mathrm{H}_{38} \mathrm{O}_{14} \mathrm{SNa}[\mathrm{M}+\mathrm{Na}]^{+}$ 665.1874 , found 665.1878 .

3.2.4 2,3,4-三- $O$-乙酰基- $\alpha-L$ - 吡喃鼠李糖- $(1 \rightarrow 3)$ 2,6 -二- $O$-乙酰基-4- $O-(3,4-O$-二烯丙氧基- $E$ - 肉桂酰 基) $-\beta$ - $D$-吡喃葡萄糖对甲苯硫酚苷(11)的合成

将化合物 9 (180 mg, $0.28 \mathrm{mmol})$, 咖啡酸片段 $\mathbf{1 0}$ (146 mg, $0.56 \mathrm{mmol}$ ), DMAP (51 mg, $0.42 \mathrm{mmol}$ )和 DCC $(145 \mathrm{mg}, 0.70 \mathrm{mmol})$ 溶于干燥的二氯甲烷 $(5 \mathrm{~mL})$ 中, 室 温反应至原料反应完全. 反应液垫硅藻土过滤, 有机相 用饱和食盐水洗, 无水 $\mathrm{Na}_{2} \mathrm{SO}_{4}$ 干燥, 过滤, 浓缩, 柱层 析 $[V$ (石油醚) $: V$ (丙酮 $)=6: 1$ 至 $4: 1$ ]得白色固体 11 (194 mg, 产率 79\%): $[\alpha]_{\mathrm{D}}^{25}-41.9$ (c 1.0, $\left.\mathrm{CHCl}_{3}\right) ;{ }^{1} \mathrm{H}$ NMR $\left(500 \mathrm{MHz}, \mathrm{CDCl}_{3}\right) \delta: 7.63(\mathrm{~d}, J=15.9 \mathrm{~Hz}, 1 \mathrm{H})$, $7.45 \sim 7.38(\mathrm{~m}, 2 \mathrm{H}), 7.13(\mathrm{~d}, J=8.0 \mathrm{~Hz}, 2 \mathrm{H}), 7.09 \sim 7.03$ $(\mathrm{m}, 2 \mathrm{H}), 6.87(\mathrm{~d}, J=8.3 \mathrm{~Hz}, 1 \mathrm{H}), 6.23(\mathrm{~d}, J=15.9 \mathrm{~Hz}$, $1 \mathrm{H}), 6.12 \sim 6.02(\mathrm{~m}, 2 \mathrm{H}), 5.46 \sim 5.40(\mathrm{~m}, 2 \mathrm{H}), 5.34 \sim 5.29$ (m, 2H), 5.19 (t, $J=9.6 \mathrm{~Hz}, 1 \mathrm{H}), 5.12(\mathrm{dd}, J=10.1,3.3$ $\mathrm{Hz}, 1 \mathrm{H}), 5.07$ (t, $J=9.6 \mathrm{~Hz}, 1 \mathrm{H}), 5.04(\mathrm{dd}, J=3.4,2.0 \mathrm{~Hz}$, $1 \mathrm{H}), 4.95$ (t, $J=10.0 \mathrm{~Hz}, 1 \mathrm{H}), 4.89$ (d, $J=2.0 \mathrm{~Hz}, 1 \mathrm{H}$ ), $4.67 \sim 4.61(\mathrm{~m}, 4 \mathrm{H}), 4.59$ (d, $J=10.0 \mathrm{~Hz}, 1 \mathrm{H}), 4.20$ (dd, $J=12.3,2.8 \mathrm{~Hz}, 1 \mathrm{H}), 4.15(\mathrm{dd}, J=12.2,5.5 \mathrm{~Hz}, 1 \mathrm{H}), 3.95$ (t, $J=9.2 \mathrm{~Hz}, 1 \mathrm{H}), 3.83$ (d, $J=3.6 \mathrm{~Hz}, 1 \mathrm{H}), 3.68$ (ddd, $J=$ 10.0, 5.5, $2.8 \mathrm{~Hz}, 1 \mathrm{H}), 2.35$ (s, 3H), $2.21(\mathrm{~s}, 3 \mathrm{H}), 2.11(\mathrm{~s}$, $3 \mathrm{H}), 2.09$ (s, 3H), 1.95 (s, 3H), $1.86(\mathrm{~s}, 3 \mathrm{H}), 1.04$ (d, $J=$ $6.2 \mathrm{~Hz}, 3 \mathrm{H}) ;{ }^{13} \mathrm{C}$ NMR $\left(126 \mathrm{MHz}, \mathrm{CDCl}_{3}\right) \delta: 170.6,170.0$, $170.0,169.5,169.4,165.5,151.1,148.6,146.4,138.4$, $133.3,133.0,132.7,129.6,128.4,127.1,123.0,118.1$, 118.0, 114.4, 113.4, 112.8, 99.0, 86.4, 81.9, 76.1, 71.3, $70.8,70.2,70.0,69.7,69.2,68.5,67.2,62.6,21.2,21.0$,
20.9, 20.8, 20.6, 20.6, 17.5; HRMS (ESI) calcd for $\mathrm{C}_{44} \mathrm{H}_{52^{-}}$ $\mathrm{O}_{17} \mathrm{SNa}[\mathrm{M}+\mathrm{Na}]^{+}$907.2817; found 907.2814.

3.2 .53 ,4-二- $O$-烯丙氧基苯乙基 $2,3,4$-三- $O$-乙酰基$\alpha-L$-吡喃鼠李糖- $(1 \rightarrow 3)-2,6$-二- $O$-乙酰基-4- $O-(3,4-$ 二$O$-烯丙氧基- $E$-肉桂酰基) $-\beta$ - $D$-吡喃葡萄糖苷(13)的合 成

将化合物 11 (210 mg, $0.24 \mathrm{mmol})$ 和 BSP (63 mg, 0.3 $\mathrm{mmol})$ 溶于二氯甲烷 $(10 \mathrm{~mL})$ 中. 将反应体系冷却至 $-60{ }^{\circ} \mathrm{C}$, 通过注射器缓慢加入三氟甲磺酸酎 $(64 \mu \mathrm{L}$, $0.36 \mathrm{mmol}$ ). $10 \mathrm{~min}$ 后, 滴加 $20 \mu \mathrm{L}$ 水. 保持 $-60{ }^{\circ} \mathrm{C}$ 反应 $20 \mathrm{~min}$ 后, $\mathrm{Et}_{3} \mathrm{~N}$ 淬灭反应. 体系升至室温, 反应液用饱 和食盐水溶液洗, 乙酸乙酯萃取两次, 合并有机相, 无 水 $\mathrm{Na}_{2} \mathrm{SO}_{4}$ 干燥, 浓缩, 快速柱层析得淡黄色糖浆.

将上述粗品, 邻-环丙乙炔基苯甲酸 $(56 \mathrm{mg}, 0.28$ $\mathrm{mmol}$ ), EDCI (54 mg, $0.28 \mathrm{mmol}$ )和 DMAP (34 mg, 0.28 $\mathrm{mmol})$ 溶于干燥的二氯甲烷 $(4 \mathrm{~mL})$ 中, 氩气保护, 通过 注射器滴加 $N, N$-二异丙基乙胺(DIPEA) $(50 \mu \mathrm{L}, 0.28$ $\mathrm{mmol}$ ), 室温反应至原料基本消耗完全. 乙酸乙酯稀释, 饱和食盐水洗，乙酸乙酯萃取两次，合并有机相，无水 $\mathrm{Na}_{2} \mathrm{SO}_{4}$ 干燥, 浓缩, 快速柱层析得浅黄色泡沫状固体.

将上述给体粗品和化合物 12 (112 mg, $0.48 \mathrm{mmol})$ 溶于干燥的二氯甲烷 $(4 \mathrm{~mL})$ 中, 加入活化好的 $4 \AA \mathrm{MS}$ $(300 \mathrm{mg})$, 氩气保护下室温搅拌 $0.5 \mathrm{~h}$. 通过注射器加入 $\mathrm{PPh}_{3} \mathrm{AuNTf}_{2}$ 的二氯甲烷溶液 $(0.05 \mathrm{~mol} / \mathrm{L}, 1 \mathrm{~mL})$, 室温下 反应至给体消耗完全. 加入 $\mathrm{Et}_{3} \mathrm{~N}$ 淬灭反应, 反应液垫硅 藻土过滤并浓缩，柱层析 $[V$ (石油醚 $): V($ 乙酸乙酯 $)=$ 4: 1 至 3: 1] 得白色泡沫状固体 13 (79 mg, 三步产率 $35 \%):[\alpha]_{\mathrm{D}}^{25}-63.2\left(c \quad 0.68, \mathrm{CHCl}_{3}\right) ;{ }^{1} \mathrm{H}$ NMR $(500 \mathrm{MHz}$, $\left.\mathrm{CDCl}_{3}\right) \delta: 7.62(\mathrm{~d}, J=15.9 \mathrm{~Hz}, 1 \mathrm{H}), 7.09 \sim 7.02(\mathrm{~m}, 2 \mathrm{H})$, $6.86(\mathrm{~d}, J=8.3 \mathrm{~Hz}, 1 \mathrm{H}), 6.81(\mathrm{~d}, J=8.2 \mathrm{~Hz}, 1 \mathrm{H}), 6.74(\mathrm{~d}$, $J=1.5 \mathrm{~Hz}, 1 \mathrm{H}), 6.71(\mathrm{~d}, J=8.1 \mathrm{~Hz}, 1 \mathrm{H}), 6.22(\mathrm{~d}, J=15.9$ $\mathrm{Hz}, 1 \mathrm{H}), 6.14 \sim 6.00(\mathrm{~m}, 4 \mathrm{H}), 5.45 \sim 5.36(\mathrm{~m}, 4 \mathrm{H}), 5.33 \sim$ $5.18(\mathrm{~m}, 5 \mathrm{H}), 5.12(\mathrm{dd}, J=10.1,3.3 \mathrm{~Hz}, 1 \mathrm{H}), 5.08(\mathrm{dd}, J=$ $9.5,8.0 \mathrm{~Hz}, 1 \mathrm{H}), 5.04 \sim 5.00(\mathrm{~m}, 1 \mathrm{H}), 4.94(\mathrm{t}, J=10.0 \mathrm{~Hz}$, $1 \mathrm{H}), 4.87(\mathrm{~d}, J=1.4 \mathrm{~Hz}, 1 \mathrm{H}), 4.67 \sim 4.54(\mathrm{~m}, 8 \mathrm{H}), 4.40(\mathrm{~d}$, $J=8.0 \mathrm{~Hz}, 1 \mathrm{H}), 4.19$ (dd, $J=12.4,4.8 \mathrm{~Hz}, 1 \mathrm{H}), 4.15(\mathrm{dd}$, $J=12.4,2.9 \mathrm{~Hz}, 1 \mathrm{H}), 4.06(\mathrm{dt}, J=9.3,6.3 \mathrm{~Hz}, 1 \mathrm{H}), 3.90(\mathrm{t}$, $J=9.4 \mathrm{~Hz}, 1 \mathrm{H}), 3.83(\mathrm{dq}, J=12.4,6.2 \mathrm{~Hz}, 1 \mathrm{H}), 3.68 \sim$ $3.57(\mathrm{~m}, 2 \mathrm{H}), 2.79(\mathrm{t}, J=6.7 \mathrm{~Hz}, 2 \mathrm{H}), 2.09(\mathrm{~s}, 3 \mathrm{H}), 2.07$ (s, $3 \mathrm{H}), 1.99$ (s, 3H), 1.94 (s, 3H), 1.84 (s, 3H), 1.03 (d, $J=$ $6.2 \mathrm{~Hz}, 3 \mathrm{H}) ;{ }^{13} \mathrm{C}$ NMR $\left(126 \mathrm{MHz}, \mathrm{CDCl}_{3}\right) \delta: 170.7,170.0$, $169.4,169.3,165.5,151.1,148.6,148.4,147.1,146.3$, $133.7,133.6,133.0,132.7,131.6,127.1,122.9,121.4$, $118.0,117.9,117.4,117.4,115.3,114.5,114.4,113.4$, $112.8,100.8,98.9,80.2,72.4,72.1,70.8,70.6,70.1,70.1$, 
$70.0,69.9,69.7,69.3,68.5,67.1,62.4,35.5,20.9,20.8$, 20.7, 20.6, 20.6, 17.5; HRMS (ESI) calcd for $\mathrm{C}_{51} \mathrm{H}_{62} \mathrm{O}_{20} \mathrm{Na}$ $[\mathrm{M}+\mathrm{Na}]^{+}$1017.3727, found 1017.3727.

$3.2 .63,4$-二-着基苯乙基 $2,3,4$-三- $O$-乙酰基- $\alpha$ - $L$-吡

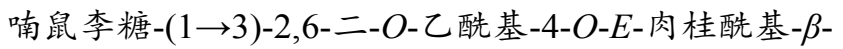
$D$-吡喃葡萄糖苷 $(14)$ 的合成

将化合物 13 (104 mg, $0.10 \mathrm{mmol})$ 溶于脱气乙酸 $(5$ $\mathrm{mL})$ 中, 氩气保护下加入 $\mathrm{Pd}\left(\mathrm{PPh}_{3}\right)_{4}(121 \mathrm{mg}, 0.10 \mathrm{mmol})$, 体系升温至 $50{ }^{\circ} \mathrm{C}$, 反应至原料消耗完全 $($ 约 $2 \mathrm{~h}$, TLC 监 测 $)$, 反应液浓缩, 柱层析 $[V$ (二氯甲烷) $: V$ (甲醇 $)=60$ : 1 至 $30: 1]$ 得浅黄色固体 $14(70 \mathrm{mg}$, 产率 $80 \%)$ : $[\alpha]_{\mathrm{D}}^{25}$ -86.2 (c 1.0, $\mathrm{CHCl}_{3}$ ); ${ }^{1} \mathrm{H}$ NMR (500 MHz, CD $\left.\mathrm{OD}\right) \delta$ : $7.62(\mathrm{~d}, J=15.9 \mathrm{~Hz}, 1 \mathrm{H}), 7.06$ (d, $J=2.1 \mathrm{~Hz}, 1 \mathrm{H}), 6.97$ $(\mathrm{dd}, J=8.2,2.1 \mathrm{~Hz}, 1 \mathrm{H}), 6.79$ (d, $J=8.1 \mathrm{~Hz}, 1 \mathrm{H}), 6.67$ (d, $J=8.0 \mathrm{~Hz}, 1 \mathrm{H}), 6.64(\mathrm{~d}, J=2.1 \mathrm{~Hz}, 1 \mathrm{H}), 6.52$ (dd, $J=8.1$, $2.0 \mathrm{~Hz}, 1 \mathrm{H}), 6.27$ (d, $J=15.8 \mathrm{~Hz}, 1 \mathrm{H}), 5.17$ (t, $J=9.6 \mathrm{~Hz}$, $1 \mathrm{H}), 5.06 \sim 5.00(\mathrm{~m}, 2 \mathrm{H}), 4.97 \sim 4.93(\mathrm{~m}, 2 \mathrm{H}), 4.90(\mathrm{t}, J=$ $9.9 \mathrm{~Hz}, 1 \mathrm{H}), 4.55(\mathrm{~d}, J=8.1 \mathrm{~Hz}, 1 \mathrm{H}), 4.21(\mathrm{dd}, J=12.3$, $4.7 \mathrm{~Hz}, 1 \mathrm{H}), 4.15 \sim 4.08(\mathrm{~m}, 2 \mathrm{H}), 4.01(\mathrm{dt}, J=9.6,6.1 \mathrm{~Hz}$, $1 \mathrm{H}), 3.87 \sim 3.78(\mathrm{~m}, 2 \mathrm{H}), 3.64(\mathrm{ddd}, J=9.6,7.8,6.6 \mathrm{~Hz}$, $1 \mathrm{H}), 2.75 \sim 2.64(\mathrm{~m}, 2 \mathrm{H}), 2.09(\mathrm{~s}, 3 \mathrm{H}), 2.06(\mathrm{~s}, 3 \mathrm{H}), 1.95$ (s, 3H), 1.92 (s, 3H), 1.80 (s, 3H), 1.02 (d, $J=6.2 \mathrm{~Hz}, 3 \mathrm{H})$; ${ }^{13} \mathrm{C}$ NMR (126 MHz, CD 3 OD) $\delta: 171.1,170.2,170.2$, $170.1,169.9,166.3,148.6,147.0,145.5,144.6,143.2$, $130.2,126.0,121.9,119.8,115.7,115.2,114.8,113.8$, $112.8,100.4,98.2,78.5,73.1,71.6,70.5,69.8,68.9,68.7$, 66.9, 62.1, 34.9, 19.5, 19.3, 19.1, 19.1, 19.0, 16.6; HRMS (ESI) calcd for $\mathrm{C}_{51} \mathrm{H}_{62} \mathrm{O}_{20} \mathrm{Na}[\mathrm{M}+\mathrm{Na}]^{+}$857.2475, found 857.2463 .

\subsubsection{Acteoside (1) 的合成}

将化合物 14 (58 mg, $0.07 \mathrm{mmol}$ )溶于二氯甲烷(2 $\mathrm{mL})$ 中, $-30{ }^{\circ} \mathrm{C}$ 下缓慢滴加质量分数为 $30 \% \sim 33 \%$ 的甲 胺甲醇溶液 $(3 \mathrm{~mL})$. 体系维持在 $-25{ }^{\circ} \mathrm{C}$ 至 $-20{ }^{\circ} \mathrm{C}$ 反应 $10 \mathrm{~h}$, 迅速浓缩除去甲胺. $\mathrm{C} 18$ 反相柱层析 $[V($ 水) $: V($ 甲 醇 $)=4: 1$ 至 $2: 1$ ] 得棕黄色固体 Acteoside (1) (18 mg, 产率 42\%): $[\alpha]_{\mathrm{D}}^{25}-69.7\left(c \quad 0.20, \mathrm{CH}_{3} \mathrm{OH}\right) ;{ }^{1} \mathrm{H}$ NMR $(500$ $\left.\mathrm{MHz}, \mathrm{CDCl}_{3}\right) \delta: 7.59(\mathrm{~d}, J=15.9 \mathrm{~Hz}, 1 \mathrm{H}), 7.05(\mathrm{~d}, J=1.9$ $\mathrm{Hz}, 1 \mathrm{H}), 6.96(\mathrm{dd}, J=1.9,8.2 \mathrm{~Hz}, 1 \mathrm{H}), 6.78(\mathrm{~d}, J=8.2 \mathrm{~Hz}$, $1 \mathrm{H}), 6.70(\mathrm{~d}, J=1.9 \mathrm{~Hz}, 1 \mathrm{H}), 6.67$ (d, $J=8.0 \mathrm{~Hz}, 1 \mathrm{H}), 6.57$ (dd, $J=1.9,8.0 \mathrm{~Hz}, 1 \mathrm{H}), 6.27$ (d, $J=15.9 \mathrm{~Hz}, 1 \mathrm{H}), 5.19$ (d, $J=1.3 \mathrm{~Hz}, 1 \mathrm{H}), 4.92$ (t, $J=9.5 \mathrm{~Hz}, 1 \mathrm{H}), 4.38$ (d, $J=7.9$ $\mathrm{Hz}, 1 \mathrm{H}), 4.08 \sim 4.02(\mathrm{~m}, 1 \mathrm{H}), 3.92(\mathrm{dd}, J=1.7,3.0 \mathrm{~Hz}$, $1 \mathrm{H}), 3.82(\mathrm{t}, J=9.2 \mathrm{~Hz}, 1 \mathrm{H}), 3.76 \sim 3.69(\mathrm{~m}, 1 \mathrm{H}), 3,57$ $(\mathrm{dd}, J=3.1,9.5 \mathrm{~Hz}, 1 \mathrm{H}), 3.65 \sim 3.50(\mathrm{~m}, 4 \mathrm{H}), 3.39(\mathrm{dd}$, $J=8.1,8.9 \mathrm{~Hz}, 1 \mathrm{H}), 3.29$ (t, $J=9.5 \mathrm{~Hz}, 1 \mathrm{H}), 2.84 \sim 2.73$ (m, 2H), 1.09 (d, $J=6.2 \mathrm{~Hz}, 3 \mathrm{H}) ;{ }^{13} \mathrm{C}$ NMR (126 MHz, $\left.\mathrm{CDCl}_{3}\right) \delta: 168.3,149.8,148.0,146.8,146.1,144.7,131.5$, $127.7,123.2,121.2,117.1,116.5,116.3,115.2,114.7$, 104.2, 103.0, 81.6, 76.2, 76.1, 73.8, 72.4, 72.3, 72.1, 70.6, 70.4, 62.4, 36.6, 18.5; HRMS (ESI) calcd for $\mathrm{C}_{29} \mathrm{H}_{36} \mathrm{O}_{15} \mathrm{Na}$ $[\mathrm{M}+\mathrm{Na}]^{+}$647.1946, found 647.1960 .

辅助材料(Supporting Information) 化合物 7-1, 7-2, 8, 9, 11，13，14 和 Acteoside (1) 的 ${ }^{1} \mathrm{H} \mathrm{NMR},{ }^{13} \mathrm{C} \mathrm{NMR}$ 和 HRMS 谱图. 这些材料可以免费从本刊网站 (http:// sioc-journal.cn/)下载.

\section{References}

[1] (a) Molgaard, P.; Ravn, H. Phytochemistry 1988, 27, 2411. (b) Park, H. J.; Jung, W. T.; Basnet, P.; Kadota, S.; Namba, T. J. Nat. Prod. 1996, 59, 1128.

[2] (a) Scarpati, M. L.; Monache, D. Ann. Chim. (Rome, Italy) 1963, 53,356 .

(b) Nonaka, G.; Nishioka, I. Phytochemistry 1977, 16, 1265. (c) Baudouin, G.; Skaltsounis, A.L.; Tillequin, F.; Koch, M. Planta Med. 1988, 54, 321.

[3] Birkofer, L.; Kaiser, C.; Thomas, U. Z. Naturforscher 1968, 23b, 1051.

[4] Leporini, L.; Menghini, L.; Foddai, M.; Petretto, G. L.; Chessa, M.; Tirillini, B.; Pintore, G. Nat. Prod. Res. 2015, 29, 899.

[5] Rao, K. Y.; Lien, H.-M.; Lin, Y.-H.; Hsu, Y.-M.; Yeh, C.-T.; Chen, C.-C.; Lai, C.-H.; Tzeng, Y.-M. Food Chem. 2012, 132, 780.

[6] (a) Inoue, M.; Sakuma, Z.; Ogihara, Y.; Saracoglu, I. Biol. Pharm. Bull. 1998, 21, 81 .

(b) Li, J.; Zheng, Y.; Zhou, H.; Su, B.; Zheng, R. Planta Med. 1997, $63,499$.

[7] Kernan, M. R.; Amarquaye, A.; Chen, J. L.; Chan, J.; Sesin, D. F.; Parkinson, N.; Ye, Z.; Barrett, M.; Bales, C.; Stoddart, C. A.; Sloan, B.; Blanc, P.; Limbach, C.; Mrisho, S.; Rozhon, E. J. J. Nat. Prod. 1998, 61, 564.

[8] Morikawa, T.; Pan, Y.; Ninomiya, K.; Imura, K.; Matsuda, H.; Yoshikawa, M.; D. Yuan D.; Muraoka, O. Bioorg. Med. Chem. 2010, $18,1882$.

[9] He, J.; Hu, X.-P.; Zeng, Y.; Li, Y.; Wu, H.-Q.; Qiu, R.-Z.; Ma, W.-J.; Li, T.; Li, C.-Y.; He, Z.-D. J. Asian Nat. Prod. Res. 2011, 13, 449.

[10] Kawada, T.; Asano, R.; Hayashida, S.; Sakuno, T. J. Org. Chem. 1999, 64, 9268.

[11] Duynstee, H. I.; de Koning, M. C.; Ovaa, H.; van der Marel, G. A.; van Boom, J. H. Eur. J. Org. Chem. 1999, 10, 2623.

[12] (a) Zhang, S.-Q.; Li, Z.-J.; Wang, A.-B.; Cai, M.-S.; Feng, R. Carbohydr. Res. 1997, 299, 281.

(b) Zhang, S.-Q.; Li, Z.-J.; Wang, A.-B.; Cai, M.-S.; Feng, R. Carbohydr. Res. 1998, 308, 281.

(c) Li, Q.; Li, S.-C.; Li, H.; Cai, M.-S.; Li, Z.-J. Carbohydr. Res. 2005, 340, 1601.

[13] Das, S. K.; Reddy, K. A.; Mukkanti, K. Carbohydr. Res. 2007, 342, 2309.

[14] Liu, Y.-G.; Li, X.; Xiong, D.-C.; Yu, B.; Pu, X.; Ye, X.-S. Eur. J. Med. Chem. 2015, 95, 313.

[15] (a) Shu, P.; Xiao, X.; Zhao, Y.; Xu, Y.; Yao, W.; Tao, J.; Wang, H.; Yao, G.; Lu, Z.; Zeng, J.; Wan, Q. Angew. Chem., Int. Ed. 2015, 54, 14432.

(b) Zhao, Y.; Zeng, J.; Liu, Y.; Xiao, X.; Sun, G.; Sun, J.; Shu, P.; Fu, D.; Meng, L.; Wan, Q. J. Carbohydr. Chem. 2018, 37, 471.

[16] (a) Khong, D. T.; Judeh, Z. M. A. Carbohydr. Res. 2016, 436, 50. (b) Khong, D. T.; Judeh, Z. Org. Biomol. Chem. 2017, 15, 2638. (c) Khong, D. T.; Judeh, Z. M. A. Tetrahedron Lett. 2017, 58, 109. 
[17] Yu, B.; Li, B.; Xing, G.; Hui, Y. J. Comb. Chem. 2001, 3, 404.

[18] (a) Yu, B. Acc. Chem. Res. 2018, 51, 507.

(b) Zhu, D.; Yu, B. Chin. J. Chem. 2018, 36, 681.

(c) Ehianeta, T. S.; Shen, D.; Xu, P.; Yu, B. Chin. J. Chem. 2019,

$37,827$.

(d) Shao, W. B.; An, Q. L.; Cao, X.; Yu, B. Acta Chim. Sinica 2019, 77, 999 (in Chinese).

(邵文博, 安泉林, 曹金金, 俞逄, 化学学报, 2019, 77, 999.)

(e) Shen, R. Z.; Cao, X.; Yu, B. Acta Chim. Sinica 2018, 76, 278 (in Chinese).

(沈仁增, 曹金金, 俞隧, 化学学报, 2018, 76, 278.)

[19] (a) Li, Y.; Yang, Y.; Yu, B. Tetrahedron Lett. 2008, 49, 3604.

(b) Yoshimura, F.; Itoh, R.; Torizuka, M.; Mori, G.; Tanino, K. Angew. Chem., Int. Ed. 2018, 57, 17161.

[20] Liang, P.-H.; Lu, Y.-J.; Tang, T.-H. Tetrahedron Lett. 2010, 51,
6928.

[21] Kong, F. Carbohydr. Res. 2007, 342, 345.

[22] Rakesh, J.; Dickman, M. H.; Kuhnert, N. Org. Biomol. Chem. 2012, $10,5266$.

[23] Hu, Z. F.; Silipo, A.; Li, W.; Molinaro, A.; Yu, B. J. Org. Chem. 2019, 84, 13733.

[24] (a) Crich, D.; Smith, M. J. Am. Chem. Soc. 2002, 124, 8867.

(b) Codée, J. D. C.; Litjens, R. E. J. N.; den Heeten, R.; Overkleeft, H. S.; van Boom, J. H.; van der Marel, G. A. Org. Lett. 2003, 5 , 1519.

[25] Xu, J.; Liu, Y.; Dupouy, C.; Chattopadhyaya, J. J. Org. Chem. 2009, $74,6534$.

[26] Kawada, T.; Asano, R.; Makino, K.; Sakuno, T. J. Wood. Sci. 2002, $48,512$.

(Cheng, F.) 